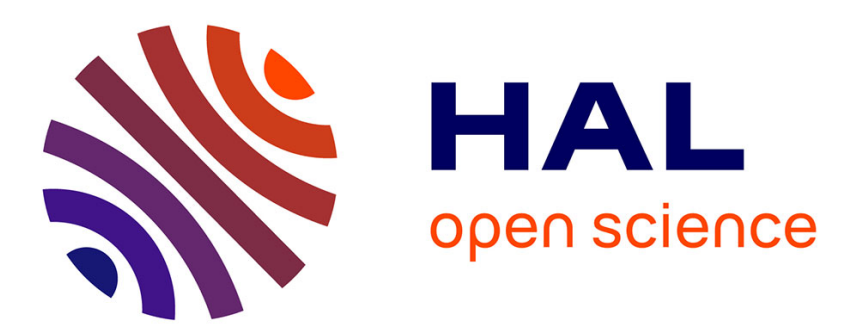

\title{
Comparative study of blades reduced order models with geometrical nonlinearities and contact interfaces
}

Elise Delhez, Florence Nyssen, Jean-Claude Golinval, Alain Batailly

\section{To cite this version:}

Elise Delhez, Florence Nyssen, Jean-Claude Golinval, Alain Batailly. Comparative study of blades reduced order models with geometrical nonlinearities and contact interfaces. ASME Turbo Expo 2020, Sep 2020, London, United Kingdom. 10.1115/GT2020-14882 · hal-02928234

\section{HAL Id: hal-02928234 \\ https://hal.science/hal-02928234}

Submitted on 2 Sep 2020

HAL is a multi-disciplinary open access archive for the deposit and dissemination of scientific research documents, whether they are published or not. The documents may come from teaching and research institutions in France or abroad, or from public or private research centers.
L'archive ouverte pluridisciplinaire HAL, est destinée au dépôt et à la diffusion de documents scientifiques de niveau recherche, publiés ou non, émanant des établissements d'enseignement et de recherche français ou étrangers, des laboratoires publics ou privés. 


\title{
Comparative study of blades reduced order models with geometrical nonlinearities and contact interfaces
}

\author{
Elise Delhez ${ }^{1,2}$, Florence Nyssen ${ }^{1}$, Jean-Claude Golinval ${ }^{2}$, Alain Batailly ${ }^{1}$
}

\begin{abstract}
This paper investigates the use of different model reduction methods accounting for geometric nonlinearities. These methods are adapted to retain physical degrees-of-freedom in the reduced space in order to ease contact treatment. These reduction methods are applied to a 3D finite element model of an industrial compressor blade (NASA rotor 37). In order to compare the different reduction methods, a scalar indicator is defined. This performance indicator allows to quantify the accuracy of the predicted displacement both locally (at the blade tip) and globally. The robustness of each method with respect to variations of the external excitation is also assessed. The performances of the reduction methods are then compared in the case of frictional contact between the blade tip and the surrounding casing. This work brings evidence that reduced order models provide a computationally efficient alternative to full order finite element models for the accurate prediction of the time response of structures with both distributed and localized nonlinearities.
\end{abstract}

Keywords

Reduced order model, geometrical nonlinearities, rotor/stator interaction

1 - Department of Mechanical Engineering, Polytechnique Montréal, P.O. Box 6079, Succ. Centre-Ville, Montréal, Québec, Canada H3C 3A7 2 - Department of Aerospace and Mechanical Engineering, University of Liège, allée de la Découverte 9, 4000 Liège, Belgium

\section{Étude comparative de modèles réduits d'aubes avec non-linéarités géométriques et interfaces de contact}

\author{
Elise Delhez ${ }^{1,2}$, Florence Nyssen ${ }^{1}$, Jean-Claude Golinval ${ }^{2}$, Alain Batailly ${ }^{1}$
}

Résumé

Cet article étudie l'utilisation de différentes méthodes de réduction de modèles tenant compte des non-linéarités géométriques. Ces méthodes sont adaptées afin de conserver des degrés de liberté physiques dans l'espace réduit et ainsi de faciliter le traitement du contact. Les méthodes de réduction sont appliquées à un modèle élément fini 3D d'une aube de compresseur industriel (l'aube rotor 37 de la NASA). Afin de comparer les différentes méthodes de réduction, un indicateur scalaire est défini. Cet indicateur de performance permet de quantifier la précision du déplacement prédit aussi bien localement (en bout d'aube) que globalement (dans toute l'aube). La robustesse de chaque méthode par rapport aux variations de l'excitation appliquée est aussi évaluée. Les performances des méthodes de réduction sont ensuite comparées dans le cas de contact avec frottement entre le bout d'aube et le carter. Ce travail montre que les modèles réduits sont des alternatives numériquement efficaces aux modèles éléments finis complets pour la prédiction précise de la réponse temporelle de structures mécaniques en présence de non-linéarités distribuées et localisées.

Mots-clés

Modèle réduit, non-linéarités géométriques, interaction rotor/stator

1 - Département de génie mécanique, Polytechnique Montréal, P.O. Box 6079, Succ. Centre-Ville, Montréal, Québec, Canada H3C 3A7 2 - Département d'aérospatiale et mécanique, Université de Liège, allée de la Découverte 9, 4000 Liège, Belgique 


\section{Introduction}

The tight weight constraints in aeronautics have direct consequences on the dynamics of aircraft structures. In particular, engine blades are now so slender and flexible that they experience large strains and displacements, yielding geometrically nonlinear behaviors $[1,2,3]$. At the same time, the need for improved engine efficiency motivates engineers to consider a reduced clearance between the rotating blades and the surrounding casing, which increases the risk of contact occurrences between rotating and stationary components in nominal operating conditions [4, 5, 6]. Nowadays, the preliminary design of turbomachines in industry in mainly driven by aerodynamic considerations and the absence of contact occurrence is only checked at the end of the process. If required, the gap between the blades and the casing is then adjusted. There is an increasing demand for the development of tools allowing to account for structural aspects in an accurate way from the beginning of the design in order to accelerate the design, but also to avoid oversizing of aircraft. In particular, a better understanding of contact dynamics is required.

In order to efficiently analyze complex industrial structures, it is necessary to build reduced order models characterized by a smaller number of parameters compared to high fidelity finite element models. While linear model order reduction techniques have been widely studied and used in the last decades [7, 8, 9], the development of nonlinear reduced order modeling techniques that allow to accelerate the computation while ensuring accurate solutions is a major concern nowadays.

When building nonlinear reduced order models with projective reduction methods, two challenges are usually encountered. The first challenge consists in finding an adequate reduction basis embedding the main nonlinear characteristics of the structure. The second challenge consists in evaluating the internal nonlinear forces in the reduced space. Different reduction bases have been recently investigated in a nonlinear context by different authors: linear bases [10, 11], bases obtained from Proper Orthogonal Decomposition (POD) [12, 3], linear bases augmented with modal derivatives (MD) $[13,14]$ and bases composed of nonlinear complex modes $[15,16]$. The methods for the determination of the reduced nonlinear forces are classified as intrusive and non-intrusive methods. In intrusive methods, reduced nonlinear forces are obtained by direct projection of the full order nonlinear forces[17, 18]. In the Discrete Empirical Interpolation Method (DEIM) [19], the Galerkin projection of the nonlinear internal forces is obtained by interpolation of the nonlinear forces computed with the high fidelity model at a given number of chosen interpolation points. However, the full order nonlinear forces cannot always be extracted from commercial finite element software. Non-intrusive methods compatible with standard finite element packages have therefore been derived. In such methods, the reduced nonlinear forces are expressed as a polynomial form of the reduced coordinates whose coefficients are identified with nonlinear static evaluations with imposed displacements (Enforced Displacement method or STiffness Evaluation Procedure (STEP) [20]) or imposed forces (Applied Force method [21]).

Different numerical strategies can be used to predict contact interactions with high fidelity finite element models, or, more efficiently, with reduced order models. Contact is usually numerically managed with penalty methods [22], with Lagrange multipliers [23] or with the augmented Lagrangian approach [24]. In the field of bladed disk dynamics, recent developments regarding the prediction of contact interactions include the modeling of a flexible casing [25], also with the extension to new materials, such as ceramic matrix composite (CMC) material [6]. The modeling of the removal of an abradable coating on the casing is also addressed in [26, 6, 5]. However, the classical methods are most of the time limited to small displacements and therefore do not account for geometrical nonlinearities.

This paper compares different nonlinear projective model order reduction methods applied to an industrial blade model. The paper is organized as follows. First, the theoretical concepts related to reduced order modeling are developed. Three different nonlinear reduction techniques, respectively the POD, the Craig-Bampton method with POD filtering of the nonlinear forces and the modal derivatives approach, are selected and adapted to allow the definition of contact interface in the reduced space. The numerical strategy used for the treatment of contact in the reduced space is also detailed. Then, a scalar indicator is defined in order to quantitatively assess the performance and the robustness of the different reduction methods. The different reduction methods are then applied to a 3D finite element model of an industrial compressor blade subjected to a harmonic excitation. The computation of the performance indicator for the different methods allows to highlight the pros and cons of each method. Contact simulations are finally conducted to assess the performance of the reduction methods in the presence of both geometrical (i.e. global) and contact (i.e. localized) nonlinearities. 


\section{Reduced order modeling}

The dynamics of mechanical structures is usually studied with high fidelity finite element models. The spatially discretized equation of motion governing the physical displacement $\mathbf{u}$ of the structure around its equilibrium state writes

$$
\mathbf{M} \ddot{\mathbf{u}}+\mathbf{C} \dot{\mathbf{u}}+\mathbf{K u}+\mathbf{g}_{n l}(\mathbf{u})=\mathbf{f}_{e}(t)+\mathbf{f}_{c}(\mathbf{u}, \dot{\mathbf{u}}),
$$

where $\mathbf{M}$ is the mass matrix, $\mathbf{C}$ is the viscous damping matrix, $\mathbf{K}$ is the linear stiffness matrix, $\mathbf{g}_{n l}(\mathbf{u})$ is the nonlinear internal forces vector, $\mathbf{f}_{e}(t)$ is the external forces vector and $\mathbf{f}_{c}(\mathbf{u}, \dot{\mathbf{u}})$ accounts for possible contact interactions. The time dependence of $\mathbf{u}$ is omitted for brevity.

The basic idea behind linear projection-based model order reduction techniques is to write the displacement field $\mathbf{u}$ as a linear combination of spatial basis functions $\boldsymbol{\Phi}$

$$
\mathbf{u}(t)=\mathbf{\Phi} \mathbf{q}(t) .
$$

The physical displacement $\mathbf{u} \in \mathbb{R}^{N}$ is therefore linearly reduced to a set of generalized coordinates $\mathbf{q} \in \mathbb{R}^{r}$, where $r \leqslant N$ (in practice $r \ll N$ ). Injecting Eqn. (2) in Eqn. (1), the projection of the equation of motion in the reduced space writes

$$
\tilde{\mathbf{M}} \ddot{\mathbf{q}}+\tilde{\mathbf{C}} \dot{\mathbf{q}}+\tilde{\mathbf{K}} \mathbf{q}+\tilde{\mathbf{g}}_{n l}(\mathbf{q})=\tilde{\mathbf{f}}_{e}(t)+\tilde{\mathbf{f}}_{c}(\mathbf{q}, \dot{\mathbf{q}}),
$$

where the projected matrices take the expressions

$$
\tilde{\mathbf{M}}=\boldsymbol{\Phi}^{\mathrm{T}} \mathbf{M} \boldsymbol{\Phi}, \quad \tilde{\mathbf{C}}=\boldsymbol{\Phi}^{\mathrm{T}} \mathbf{C} \boldsymbol{\Phi}, \quad \tilde{\mathbf{K}}=\boldsymbol{\Phi}^{\mathrm{T}} \mathbf{K} \boldsymbol{\Phi}
$$

and the projected vectors are

$$
\tilde{\mathbf{g}}_{n l}(\mathbf{q})=\boldsymbol{\Phi}^{\mathrm{T}} \mathbf{g}_{n l}(\boldsymbol{\Phi} \mathbf{q}), \tilde{\mathbf{f}}_{e}=\boldsymbol{\Phi}^{\mathrm{T}} \mathbf{f}_{e}, \tilde{\mathbf{f}}_{c}(\mathbf{q}, \dot{\mathbf{q}})=\boldsymbol{\Phi}^{\mathrm{T}} \mathbf{f}_{c}(\boldsymbol{\Phi} \mathbf{q}, \boldsymbol{\Phi} \dot{\mathbf{q}}) .
$$

It should be noted that this reduction procedure conserves the general expression of the equation of motion so that the same time integration algorithms as those used for the full order model can be used.

These reduction methods differentiate between an expensive offline stage where the projection basis is computed and the reduced order model is built and an efficient online stage where the problem is solved. The different nonlinear reduction methods differ by the choice of the projection basis $\boldsymbol{\Phi}$ and the way the projected nonlinear term $\tilde{\mathbf{g}}_{n l}(\mathbf{q})$ is evaluated. The two following sections detail the strategies adopted for solving these two issues. Then, the numerical strategy adopted for the modeling of contact in the reduced space is described.

\section{Computation of the reduced nonlinear terms}

The direct computation of the reduced nonlinear forces $\tilde{\mathbf{g}}_{n l}(\mathbf{q})$ with Eqn. (5) is not suitable for two main reasons. First, the computational complexity of the nonlinear term evaluation still depends on the total number of degrees-offreedom. Then, this way of proceeding is intrusive because it requires to have access to the full order internal forces, and therefore to the source code of the finite element software. In order to be compatible with commercial finite element software, this paper focuses on non-intrusive methods. In particular, the STiffness Evaluation Procedure (STEP) is chosen here because it has proven to be efficient for the modeling of dynamical mechanical systems subjected to large displacements [20, 27, 10, 28].

In the STEP method, the nonlinear reduced term is assumed to be a third degree polynomial in terms of the generalized coordinates $q_{1}, q_{2}, \ldots, q_{r}$, where $r$ is the number of modes in the reduction basis. The $r$ components of the vector $\tilde{\mathbf{g}}_{n l}(\mathbf{q})$ are therefore approximated by

$$
\tilde{g}_{n l}^{m}(\mathbf{q})=\sum_{i=1}^{r} \sum_{j=i}^{r} \tilde{A}_{i j}^{m} q_{i} q_{j}+\sum_{i=1}^{r} \sum_{j=i}^{r} \sum_{k=j}^{r} \tilde{B}_{i j k}^{m} q_{i} q_{j} q_{k} .
$$

The identification of the tensors $\tilde{\mathbf{A}}$ and $\tilde{\mathbf{B}}$ requires $\left(r^{3}+6 r^{2}+5 r\right) / 6$ nonlinear forces static evaluations performed with the high fidelity finite element model with imposed displacements. The identification procedure is summarized in [20]. 


\section{Reduction basis}

Three different reduction bases are investigated in this paper, respectively obtained with the POD, Craig-Bampton and modal derivatives approaches. In order to keep the physical contact interface in the reduced space, and therefore to allow the implementation of contact laws directly in the reduced space, the different reduction bases are (if required) adapted to retain the corresponding physical degrees-of-freedom in the reduced coordinates. The physical displacement vector $\mathbf{u}$ is therefore partitioned into its inner degrees-of-freedom and boundary interface degrees-of-freedom counterparts, $\mathbf{u}_{i}$ and $\mathbf{u}_{b}$. The boundary interface degrees-of-freedom should be included in the reduced basis. The structural matrices $\mathbf{M}, \mathbf{C}$ and $\mathbf{K}$ and the force vectors $\mathbf{g}_{n l}, \mathbf{f}_{e}$ and $\mathbf{f}_{c}$ are partitioned accordingly with respect to inner (subscript $i$ ) and boundary (subscript $b$ ) degrees-of-freedom.

\section{Proper Orthogonal Decomposition}

The model reduction by POD is a data-driven method that identifies the optimal subspace from a set of displacement snapshots obtained from simulations of the full order model [12]. If the $m$ displacement snapshots are denoted by $\mathbf{u}_{s}\left(t_{i}\right)(i=1, \ldots, m)$ and collected in a matrix

$$
\mathbf{X}=\left[\begin{array}{llll}
\mathbf{u}_{s}\left(t_{1}\right) & \mathbf{u}_{s}\left(t_{2}\right) & \cdots & \mathbf{u}_{s}\left(t_{m}\right)
\end{array}\right]
$$

the principle of the POD reduction method consists in finding the subspace $\boldsymbol{\Phi}_{s}$ such that the projection of the data matrix $\mathbf{X}$ onto $\boldsymbol{\Phi}_{s}$ has maximal spatial variance. In practice, the POD basis is obtained by identifying the dominant left singular vectors of the Singular Value Decomposition (SVD) of the data matrix $\mathbf{X}$ corresponding to the highest singular values.

In its general formulation, the POD method does not allow to retain physical degrees-of-freedom in the generalized coordinates q. In order to ease the implementation of contact interfaces, it is proposed to adapt this reduction method by defining the projection basis $\boldsymbol{\Phi}$ as a combination of $r_{b}$ constraint modes and $r_{\mathrm{POD}}$ POD modes.

The constraint modes, or Guyan modes, correspond to the linear static deformation of the structure to unit displacements at the boundary degrees-of-freedom. They can be written as

$$
\Psi=\left[\begin{array}{c}
\mathbf{\Psi}_{i} \\
\mathbf{I}
\end{array}\right]
$$

where $\boldsymbol{\Psi}_{i}$ is the solution of

$$
\mathbf{K} \Psi=\left[\begin{array}{ll}
\mathbf{K}_{i i} & \mathbf{K}_{i b} \\
\mathbf{K}_{b i} & \mathbf{K}_{b b}
\end{array}\right]\left[\begin{array}{c}
\mathbf{\Psi}_{i} \\
\mathbf{I}
\end{array}\right]=\left[\begin{array}{c}
\mathbf{0} \\
\mathbf{R}
\end{array}\right],
$$

which gives

$$
\mathbf{\Psi}_{i}=-\mathbf{K}_{i i}^{-1} \mathbf{K}_{i b}
$$

The POD modes are computed in the same way as described previously. According to the degrees-of-freedom partitioning, they write

$$
\mathbf{\Phi}_{s}=\left[\begin{array}{l}
\mathbf{\Phi}_{s, i} \\
\mathbf{\Phi}_{s, b}
\end{array}\right] .
$$

The interface component of the POD basis is not null since the interface is not set fixed. Since the interface motion is already fully represented by the constraint modes, the POD vectors are modified to fixed-interface modes by subtracting the component already present in the constraint modes

$$
\left[\begin{array}{c}
\boldsymbol{\Phi}_{\mathrm{POD}} \\
\mathbf{0}
\end{array}\right]=\left[\begin{array}{c}
\boldsymbol{\Phi}_{s, i} \\
\boldsymbol{\Phi}_{s, b}
\end{array}\right]-\left[\begin{array}{c}
\boldsymbol{\Psi}_{i} \\
\mathbf{I}
\end{array}\right] \boldsymbol{\Phi}_{s, b} .
$$

The reduction basis therefore writes

$$
\boldsymbol{\Phi}=\left[\begin{array}{cc}
\mathbf{\Psi}_{i} & \boldsymbol{\Phi}_{\mathrm{POD}} \\
\mathbf{I} & \mathbf{0}
\end{array}\right]
$$




\section{Craig-Bampton basis}

The Craig-Bampton reduction method has been widely applied in the linear context [29]. In this method, the projection basis $\boldsymbol{\Phi}$ is composed of $r_{c}$ fixed interface linear normal modes $\boldsymbol{\Theta}$ and $r_{b}$ constraint modes $\boldsymbol{\Psi}$. The fixed interface linear normal modes correspond to the modes of the structure clamped at the boundary interface degrees-of-freedom. They can be written as

$$
\boldsymbol{\Theta}=\left[\begin{array}{c}
\boldsymbol{\Theta}_{i, r_{c}} \\
\mathbf{0}
\end{array}\right],
$$

where $\boldsymbol{\Theta}_{i, r_{c}}$ is formed by truncating the solution $\boldsymbol{\Theta}_{i}$ of the eigenvalue problem

$$
\mathbf{K}_{i i} \Theta_{i}=\mathbf{M}_{i i} \Theta_{i} \omega^{2}
$$

to the first $r_{c}$ modes. The constraint modes are defined by Eqn. (8). The reduction basis therefore writes

$$
\boldsymbol{\Phi}=\left[\begin{array}{cc}
\boldsymbol{\Psi}_{i} & \boldsymbol{\Theta}_{i, r_{c}} \\
\mathbf{I} & \mathbf{0}
\end{array}\right]
$$

The projection of nonlinear forces onto a linear basis (e.g. basis composed of linear vibration modes or CraigBampton basis) to obtain the generalized forces in the reduced order model may create spurious artifacts in the time response, inducing lower amplitude displacement with higher harmonics. To avoid such behavior, Balmaseda et al. [10] suggest to carry out a filtering of the full order model nonlinear forces. In practice, the nonlinear forces are collected from a given number of snapshots that represent a set of characteristic displacements in the response $\mathbf{u}_{1}, \ldots, \mathbf{u}_{m}$

$$
\mathbf{X}=\left[\begin{array}{llll}
\mathbf{g}_{n l}\left(\mathbf{u}_{1}\right) & \mathbf{g}_{n l}\left(\mathbf{u}_{2}\right) & \cdots & \mathbf{g}_{n l}\left(\mathbf{u}_{m}\right)
\end{array}\right] .
$$

The nonlinear basis $\boldsymbol{\Phi}_{f}$ used for the filtering is obtained by implementing the SVD of matrix $\mathbf{X}$. The nonlinear basis consists in the truncation to $r_{f}$ modes in the resulting left singular vectors of the SVD basis. The nonlinear forces in the full order model are then filtered with this basis $\boldsymbol{\Phi}_{f}$ and the resulting forces are used as an input to the STEP method.

\section{Craig-Bampton basis enhanced with modal derivatives}

The main limitation of extending existing linear reduction methods to a nonlinear framework lies in the fact that modal properties, and in particular vibration modes, are no longer constant but vary with the amplitude of the motion. Enriching linear bases with modal derivatives was first introduced as a way of intrinsically accounting for the nonlinear behavior of the structure in linear bases composed of linear normal modes [30]. The concept has then been recently extended to other linear reduction bases, and in particular to the classical Craig-Bampton basis [31]. In a nonlinear context, modes depend on the deformation of the structure. Let's denote by $\tilde{\boldsymbol{\Phi}}(\mathbf{u})$ the Craig-Bampton modes computed at a certain level of deformation $\mathbf{u}$. In practice, these modes are computed in the same way as the modes $\boldsymbol{\Phi}$ in Eqn. (16) by using the tangent stiffness matrix

$$
\mathbf{K}_{T}=\mathbf{K}+\frac{\partial \mathbf{g}_{n l}}{\partial \mathbf{u}}
$$

instead of the stiffness matrix K in Eqn. (10) and Eqn. (15). Modal derivatives write

$$
\psi_{i j}=\left.\frac{\partial \tilde{\phi}_{i}}{\partial q_{j}}\right|_{\mathbf{q}=\mathbf{0}} \quad i, j=1, \cdots, r,
$$


where $\tilde{\phi}_{i}$ stands for the $i^{\text {th }}$ mode of the reduction basis $\tilde{\boldsymbol{\Phi}}$ and $q_{j}$ is the $j^{\text {th }}$ generalized coordinate, see Eqn. (2). Modal derivatives describe the variation of the linear modes $\phi_{i}$ when the structure is deformed in the shape of another mode. In practice, modal derivatives are computed by solving the linear systems

$$
\mathbf{K} \psi_{i j}=-\left.\frac{\partial \mathbf{K}_{T}}{\partial q_{i}}\right|_{\mathbf{q}=\mathbf{0}} \phi_{j} .
$$

It can be shown that modal derivatives are symmetric [13], i.e.

$$
\boldsymbol{\psi}_{i j}=\boldsymbol{\psi}_{j i}
$$

so that a basis consisting in $r$ linear modes can be enhanced with $r(r+1) / 2$ modal derivatives.

The size of the reduction basis therefore increases quadratically with the number of linear modes. However, it is expected that only a few modal derivatives are necessary to capture the nonlinear behavior of the structure [32]. A selection criterion is therefore defined in order to select the best modal derivatives. The basic idea is to associate a weight to all modal derivatives according to their contribution and to select the modal derivatives characterized by the highest weights. The weight is defined by the modal interaction between the modes during a linear simulation

$$
W_{i j}=\int_{0}^{T}\left|q_{i}(t) q_{j}(t)\right| d t
$$

where $W_{i j}$ represents the weight associated to the modal derivative $\psi_{i j}$ and $q_{i}(t)$ is the time varying amplitude of the $i^{\text {th }}$ linear mode obtained in the response to a given applied external loading. While the computation of the modal derivatives is a simulation-free procedure, i.e. a procedure that does not require the time integration of the full order equation of motion (contrary to the POD method for instance), the selection of the modal derivatives requires a linear simulation of the system, which is a computationally cheap numerical time integration. This selection criterion has proven to be efficient to select modal derivatives of linear normal modes [30]. It is here proposed to apply this selection criterion for the selection of modal derivatives of Craig-Bampton modes.

\section{Contact modeling}

Model order reduction by projection conserves the general form of the equation of motion. Moreover, the three adapted reduction techniques introduced above allow to retain the physical contact interface in the reduced space. The usual contact algorithms defined for high fidelity finite element models can therefore be directly applied in the reduced space.

The computation is made in the time domain in order to capture the transient dynamics of the structure due to the occurrence of contact events. In practice, the numerical strategy adopted in this paper relies on the explicit central difference time integration scheme with the handling of contact using Lagrange multipliers [23]. Friction is managed with the Coulomb law.

At each time step $t_{n+1}$, the displacement field is first predicted without accounting for possible contact interaction as

$$
\begin{aligned}
\mathbf{q}_{n+1}^{\star}= & (2 \tilde{\mathbf{M}}+h \tilde{\mathbf{C}})^{-1}\left\{2 h^{2}\left[\tilde{\mathbf{f}}_{e}\left(t_{n}\right)-\tilde{\mathbf{g}}_{n l}\left(\mathbf{q}_{n}\right)\right]\right. \\
& \left.+\left(4 \tilde{\mathbf{M}}-2 h^{2} \tilde{\mathbf{K}}\right) \mathbf{q}_{n}+(h \tilde{\mathbf{C}}-2 \tilde{\mathbf{M}}) \mathbf{q}_{n-1}\right\},
\end{aligned}
$$

where $h$ is the time step of integration. Then, the gap between the impacting nodes and the target surface is computed. If a penetration is detected, the contact forces $\tilde{\mathbf{f}}_{c, n+1}$ are computed in the normal and tangential directions through the use of Lagrange multipliers using the methodology described in [23]. The displacement field is finally corrected to avoid penetration by

$$
\mathbf{q}_{n+1}=\mathbf{q}_{n+1}^{\star}+\left(\frac{\tilde{\mathbf{M}}}{h^{2}}+\frac{\tilde{\mathbf{C}}}{2 h}\right)^{-1} \tilde{\mathbf{f}}_{c, n+1} .
$$




\section{Performance indicator}

In order to assess the performance of the reduction methods and allow their comparison, a scalar performance indicator $I$ is defined. The performance indicator is defined as a weighted sum of $N_{c}$ different criteria as

$$
I=\sum_{i=1}^{N_{c}} w_{i} e_{i}
$$

Each criterion $e_{i}$ varies between 0 (worst case) and 1 (perfect correspondance between the solutions obtained with the full and reduced order models). The sum of the weighting factors $w_{i}$ is equal to 1 . The relative magnitudes of the weighting factors reflect the relative influence of the different criteria in the performance indicator definition. The performance indicator therefore also varies between 0 and 1 , the higher the performance indicator, the better the reduction method.

Four criteria are here selected in order to account for the global and local differences between the time solutions obtained with the full order finite element model $\mathbf{u}^{\mathrm{FOM}}(t)$ and the reduced order model $\mathbf{u}^{\mathrm{ROM}}(t)$ :

- the Covariance Criterion at maximal force amplitude

$$
e_{1}=\frac{\left[\mathbf{x}_{1}^{\mathrm{T}} \mathbf{x}_{2}\right]^{2}}{\left[\mathbf{x}_{1}^{\mathrm{T}} \mathbf{x}_{1}\right]\left[\mathbf{x}_{2}^{\mathrm{T}} \mathbf{x}_{2}\right]},
$$

where $\mathbf{x}_{1}=\mathbf{u}^{\mathrm{FOM}}\left(t_{\max }\right), \mathbf{x}_{2}=\mathbf{u}^{\mathrm{ROM}}\left(t_{\max }\right)$ and $t_{\max }$ corresponds to the time when the excitation reaches its first maximal value,

- the relative error on the energy (defined as the sum of the kinetic energy and the linear energy of deformation)

$$
e_{2}^{\prime}=\frac{\sqrt{\sum_{t}[\Delta E(t)]^{2}}}{\sqrt{\sum_{t}\left[E^{\mathrm{FOM}}(t)\right]^{2}}},
$$

where

$$
E(t)=\frac{1}{2} \dot{\mathbf{u}}(t)^{\mathrm{T}} \mathbf{M} \dot{\mathbf{u}}(t)+\frac{1}{2} \mathbf{u}(t)^{\mathrm{T}} \mathbf{K} \mathbf{u}(t)
$$

and

$$
\Delta E(t)=E^{\mathrm{FOM}}(t)-E^{\mathrm{ROM}}(t),
$$

- the relative oscillations deviation at blade tip

$$
e_{3}^{\prime}=\sqrt{\frac{1}{3 N_{\text {tip }}} \sum_{i \in \text { index }_{\text {tip }}}\left(\frac{o_{i}^{\mathrm{FOM}}-o_{i}^{\mathrm{ROM}}}{o_{i}^{\mathrm{FOM}}}\right)^{2}},
$$

where $N_{\text {tip }}$ is the number of nodes located at blade tip, index tip $_{\text {are }}$ the indices corresponding to the degreesof-freedom of these blade tip nodes and

$$
o_{i}=\max \left[\mathbf{u}_{i}(t)\right]-\min \left[\mathbf{u}_{i}(t)\right],
$$

- the relative error on the displacement at the blade tip nodes

$$
e_{4}^{\prime}=\frac{\sqrt{\sum_{t} \Delta \mathbf{u}_{b}(t)^{\mathrm{T}} \Delta \mathbf{u}_{b}(t)}}{\sqrt{\sum_{t} \mathbf{u}_{b}^{\mathrm{FOM}}(t)^{\mathrm{T}} \mathbf{u}_{b}^{\mathrm{FOM}}(t)}},
$$

where

$$
\Delta \mathbf{u}_{b}(t)=\mathbf{u}_{b}^{\mathrm{FOM}}(t)-\mathbf{u}_{b}^{\mathrm{ROM}}(t) .
$$


The second, third and fourth criteria are modified as

$$
e_{j}=1-e_{j}^{\prime} \quad(j=2,3,4)
$$

so that unitary values of the criteria correspond to a perfect matching of the solutions. Negative values of the criteria $e_{j}(j=1,2,3,4)$ are set to 0 when computing the performance indicator $I$. The first two criteria are global criteria: they allow to account for the differences in shape and in energy between the solutions obtained with the full order and the reduced order models in the entire blade. The third criterion quantifies the error on the peak-to-peak amplitude of the oscillations at blade tip. The fourth criterion quantifies the error on the amplitude of the displacement at the blade tip nodes. These criteria allow to account specifically for the local error at the tip. The local criteria are particularly important when considering contact simulations as contact will occur at blade tip. The weighting factors are defined such that global factors (and therefore local factors) account for $50 \%$ $\left(w_{1}=w_{2}=w_{3}=w_{4}=0.25\right)$.

\section{Case study}

The specific structure selected for this comparative study is the NASA rotor 37 blade. Rotor 37 is a transonic axial flow compressor stage initially designed and tested at NASA's Lewis Research Center in the late 70s [33, 34]. Since then, NASA rotor 37 has been often used as a benchmark to validate numerical methods in aerodynamics [35]. Recently, the idea of using NASA rotor 37 as a benchmark in structural dynamics, and more precisely for contact simulations, has emerged [5]. The establishment of this benchmark comes with a detailed description of the blade, with all necessary geometry parameters and material properties, which is used in this work.

Rotor 37 is made of 200-grade maraging steel with density $\rho=8,000 \mathrm{~kg} / \mathrm{m}^{3}$, Young's modulus $E=180 \mathrm{GPa}$ and Poisson's ratio $\nu=0.3$. Each blade is $7 \mathrm{~cm}$ high. The spatial discretization of the blade is shown in Fig. 1 . The finite element model has 17,235 degrees-of-freedom. In the simulations, the blade is supposed to be clamped at its root (red surface in Fig. 1). Regarding the modeling of damping, the assumption of proportional damping is made. As suggested in [5], a modal damping $\xi=5 \cdot 10^{-4}$ is considered for the first bending mode.

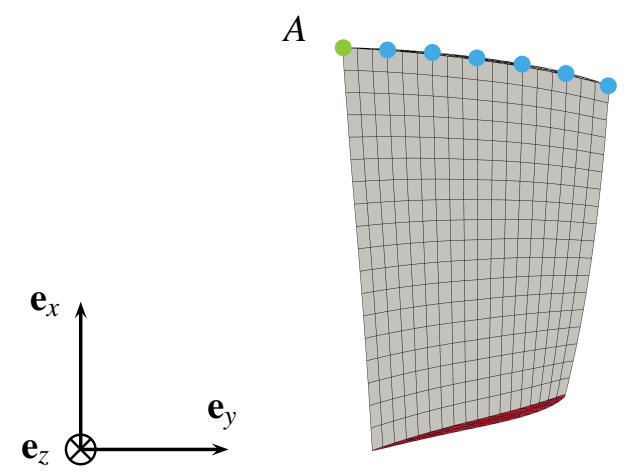

Figure 1. Test case with mesh used for the simulations and clamped surface ( $\mathbf{\square})$. The colored nodes are the blade tip nodes used for the definition of the excitation and the contact interface.

\section{Offline stage: construction of the reduced models}

The different nonlinear reduction methods are applied to the structure, with different sizes $r$ of the reduction basis. The boundary degrees-of-freedom kept in the reduced space are defined as the 3 degrees-of-freedom corresponding to the node $A$ at the blade tip trailing edge, see Fig. 1. The characteristics of the different reduction bases are summarized in Tab. 1.

For the POD reduction method, the reduction basis is composed of 3 constraint modes and $(r-3)$ POD modes. The displacement snapshots used in the POD method are obtained by computing the response of the structure to a training excitation with the high fidelity model. The blade is here excited at one node at blade tip (node $A$ in 


\begin{tabular}{rcccc}
\hline & $r_{b}$ & $r_{c}$ & $r_{\mathrm{MD}}$ & $r_{\mathrm{POD}}$ \\
\hline POD & 3 & $/$ & $/$ & $r-3$ \\
CB with POD correction & 3 & $r-3$ & $/$ & $/$ \\
CB and MD & 3 & 4 & $r-7$ & $/$ \\
\hline
\end{tabular}

Table 1. Characteristics of the reduced bases.

Fig. 1) in the $\mathbf{e}_{z}$ direction with a harmonic excitation during one period. The harmonic excitation is characterized by an amplitude of $300 \mathrm{~N}$ and a pulsation of 2,000 rad/s. Fig. 2 compares the linear and nonlinear responses of the structure at node $A$, obtained by time integration of the equation of motion (1) with the $\alpha$-generalized integration scheme (in practice using the finite element software Samcef). The high amplitude of the excitation allows to excite nonlinearities in the structure, as differences of up to $25 \%$ are observed between the linear and nonlinear displacement fields of the structure (see ). Given that the first two natural frequencies of the clamped blade are equal to $5,272 \mathrm{rad} / \mathrm{s}$ (first bending mode) and 15,760 rad/s (first torsion mode) respectively, the excitation frequency allows to mainly excite the first mode of the structure.

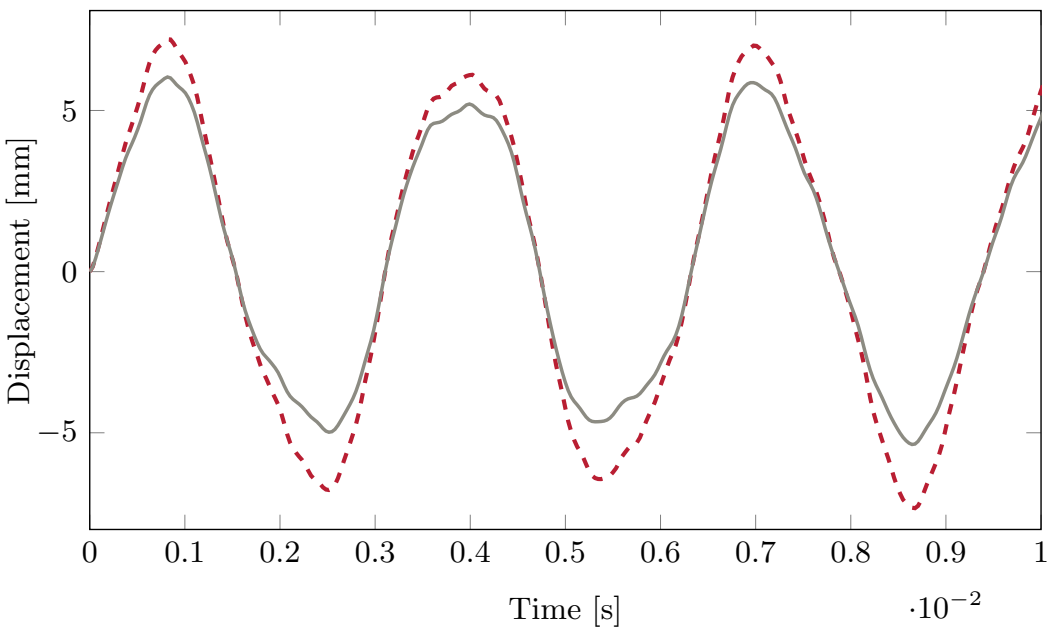

Figure 2. Linear (-- - ) and nonlinear (-) responses of the blade at node $A$ to the training excitation (extended to 3 periods).

The Craig-Bampton basis is built with 3 constraint modes and $(r-3)$ fixed interface modes. The same training excitation is used for the POD filtering of the nonlinear internal forces.

The modal derivative basis is built with 3 constraint modes, 4 fixed interface modes and $(r-7)$ modal derivatives selected according to the criteria defined by Eqn. (22). Figure 3 shows the weights $W_{i j}$ associated to the different modal derivatives for the same training excitation. The weights have been raised to the power of 0.25 to better show the relative contributions of the modes. This figure also highlights the 10 modal derivatives with the highest weights (blue bars in the figure). The dominating modal derivatives correspond to the modal derivatives of the static modes with respect to the static modes themselves. Then, the modal derivatives of the static modes with respect to the first internal modes have to be selected. It should be noted that it is not guaranteed that the modal derivatives are orthogonal and linearly independent. The modal derivatives are therefore orthonormalized with a modified Gram-Schmidt procedure which also allows to remove the linearly dependent vectors from the basis. This transformation allows to get better results and to accelerate the convergence of the nonlinear time integration scheme. 


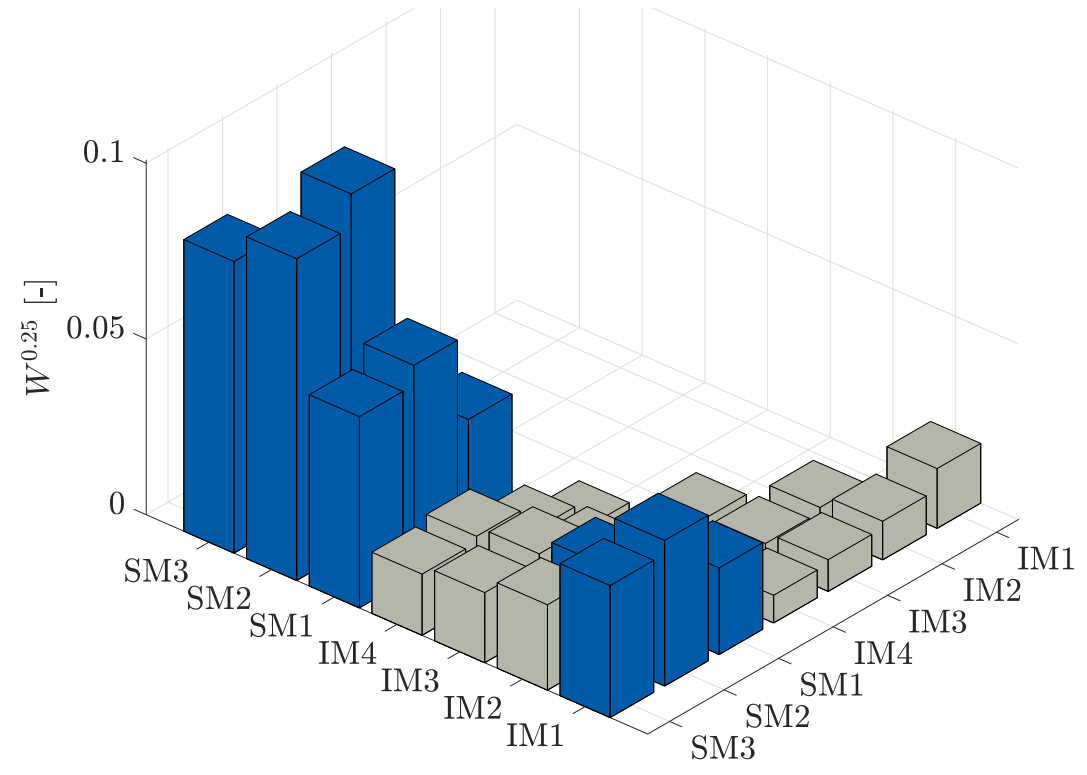

Figure 3. Weights $W_{i j}$ associated to each modal derivative (10 highest weights $(\mathbf{\square})$ ).

\section{Online stage: simulations without contact}

As a first step, the performances of the three reduction methods are assessed without contact interactions. The equation of motion (3) is numerically integrated using the $\alpha$-generalized integration scheme where numerical damping is added to stabilize the modes of numerical origin in the high frequency range.

\section{Modal derivatives selection}

Before comparing the reduction methods, the relevance of the criterion adopted for the modal derivatives selection is underlined, based on the studied test case. The time responses of the structure are computed with modal derivatives reduction bases. The external forcing is defined as the excitation for which the bases have been initially built, i.e. a harmonic excitation with amplitude of $300 \mathrm{~N}$ and pulsation of 2,000 rad/s applied at node $A$.

Figure 4 shows the time response of the structure with the reduced basis composed of the 10 selected modal derivatives highlighted in Fig. 3 and compares it to the solutions obtained with bases composed of 10 randomly chosen modal derivatives and composed of all the 28 modal derivatives associated to the 7 Craig-Bampton modes. This figure highlights that keeping only 10 modal derivatives in the reduction basis instead of 28 does not alter significantly the accuracy of the reduction basis provided that the choice of the modal derivatives is made carefully with a pertinent indicator. Choosing randomly the 10 modal derivatives provides poor results.

The different modal derivatives reduction bases can also be compared with the performance indicator $I$ defined by Eqn. (25). When all modal derivatives are included in the reduced basis, $I=0.96$. When the best 10 modal derivatives are included, $I=0.93$. For the 10 modal derivatives randomly chosen, $I=0.75$. These values reflect the qualitative comparison based on Fig. 4 and give confidence in the use of the modal derivatives selection indicator for the next studies.

\section{Reduction methods performance}

The performances of the reduction methods are assessed with the performance indicator. The external excitation is defined as the training excitation, i.e. as the excitation used for the construction of the POD reduction basis, for the POD filtering of the nonlinear forces in the Craig-Bampton method and for the selection of the modal derivatives. The harmonic excitation is characterized by an amplitude $A=300 \mathrm{~N}$, a pulsation $\omega=2,000 \mathrm{rad} / \mathrm{s}$ and is applied at $N=1$ node $($ node $A)$. 


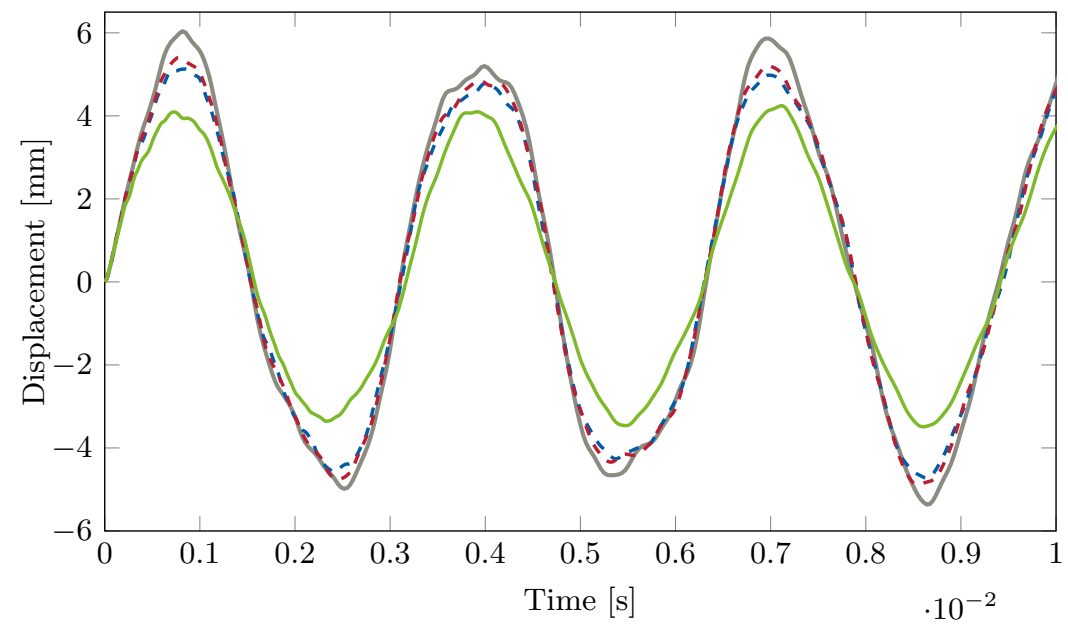

Figure 4. Comparison of the reference nonlinear solution (_-) and the solutions obtained with all modal derivatives (- - - ), with the best $10(---)$ and 10 randomly chosen (-) modal derivatives.

The response of the structure is computed for each reduction method, for different sizes of the reduction bases, and the performance indicator is evaluated for each case. Figure 5 shows the evolution of the performance indicator for each reduction methods with the number $r$ of vectors in the reduction basis. The reduction bases built by POD clearly outperform the other reduction methods in this particular case. Very good results are obtained with small reduction basis size; the indicator performance takes values very close to 1 when $r$ is larger than 10 . The Craig-Bampton basis with POD filtering and the modal derivative approach give similar results. Smaller reduction bases are required with the Craig-Bampton method to provide satisfying results (performance index larger than 0.9 ) but the modal derivative approach allows to reach slightly higher performance indicator. For large size of the reduction basis, the difference in performance criteria is reflected in the global criteria: reduction bases with modal derivatives allow a better representation of the global behavior of the blade dynamics.

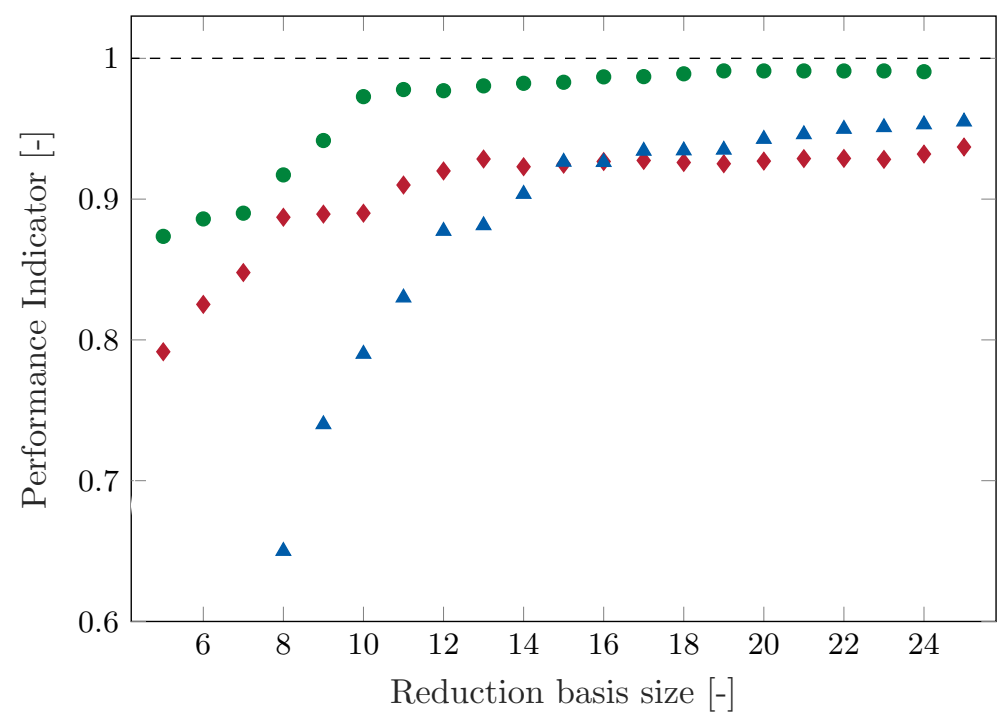

Figure 5. Evolution of the performance indicator for the different reduction methods (POD (•), Craig-Bampton with POD filtering ( $)$, modal derivatives $(\boldsymbol{\Delta}))$ as a function of the size of the reduced basis $(A=300 \mathrm{~N}, \omega=2,000 \mathrm{rad} / \mathrm{s}, N=1)$. 


\section{Reduction methods robustness}

The robustness of each reduction method with respect to a variation of the external excitation is then assessed using the same performance indicator. The reduction bases used in Fig. 5 are reused to compute the response of the structure to a harmonic excitation of amplitude $A=300 \mathrm{~N}$ and pulsation $\omega=1,000 \mathrm{rad} / \mathrm{s}$. The performance indicator is computed for the different methods, for each reduction basis size. The results are presented in Fig. 6. The performances of the different reduction methods do not seem to be affected when another excitation pulsation is considered. The relative performance of the methods is also conserved: the POD method provides the best results. The modal derivative method provides better results than the Craig-Bampton method with POD filtering when large reduction basis are considered. The study of the robustness of the methods to change in the amplitude of the external excitation leads to similar conclusions.

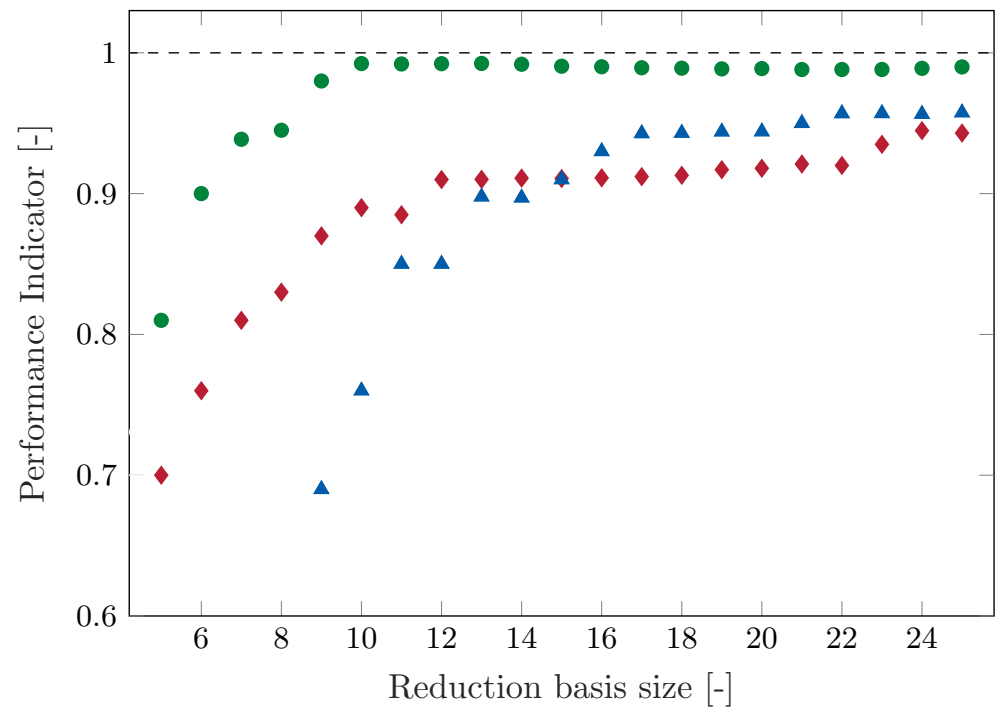

Figure 6. Evolution of the performance indicator for the different reduction methods (POD ( $\bullet$ ), Craig-Bampton with POD filtering $(\bullet)$, modal derivatives $(\boldsymbol{\Delta}))$ as a function of the size of the reduced basis $(A=300 \mathrm{~N}, \omega=1,000 \mathrm{rad} / \mathrm{s}, N=1)$.

The robustness of the methods with respect to variation of the spatial shape of the external excitation is then assessed. The reduction bases are used to compute the response of the structure to a harmonic excitation of amplitude $A=300 \mathrm{~N}$ and pulsation $\omega=2,000 \mathrm{rad} / \mathrm{s}$ distributed on the $N=7$ blade tip nodes represented in Fig. 1. The results are presented in Fig. 7. The performance of the POD method clearly drops when a different spatial shape of the excitation is considered. The performance of the Craig-Bampton method with POD filtering is also slightly decreased. The performance of the modal derivative method remains approximately the same. The modal derivative approach appears therefore as the most robust reduction method among those analyzed here.

\section{Online stage: simulations with contact}

The performances of the reduction methods are then assessed when contact interactions occur between the blade tip and the rigid casing. In practice, only contact interaction at node $A$ is considered in this study. At the beginning of the simulation, the casing is perfectly circular to avoid any initial penetration. In order to initiate contact, the casing is progressively deformed in the radial direction until reaching the final distortion

$$
f(\theta)=h_{b} \exp \left[-\left(\frac{\theta / \pi-\lfloor\theta / \pi\rfloor}{0.15}\right)^{2}\right]
$$

where $\theta$ is the angular coordinate varying between 0 and $2 \pi$. This distortion corresponds to an ovalization of the casing with the creation of two diametrically opposed bumps of height $h_{b}$ where contact can occur. 


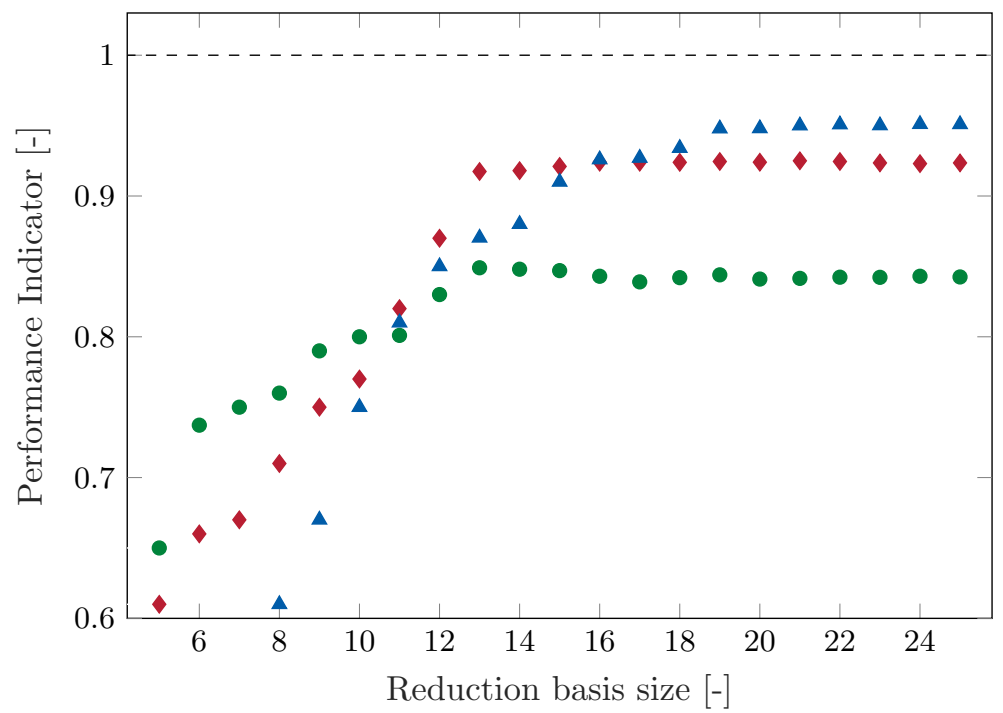

Figure 7. Evolution of the performance indicator for the different reduction methods (POD ( $\bullet$ ), Craig-Bampton with POD filtering $(\downarrow)$, modal derivatives $(\boldsymbol{\Delta}))$ as a function of the size of the reduced basis $(A=300 \mathrm{~N}, \omega=2,000 \mathrm{rad} / \mathrm{s}, N=7)$.

The rotation speed is fixed to $\Omega=1,000 \mathrm{rad} / \mathrm{s}$. Friction is modeled using a Coulomb law with a friction coefficient $\mu=0.15$. The initial clearance between the casing and the blade tip is set to $0.356 \mathrm{~mm}$, as for the original rotor 37 [33]. The bump height is fixed to $1.5 \mathrm{~mm}$. This bump height allows to activate the geometrical nonlinearities of the structure. As shown in Fig. 8, a significant difference between the evolutions of the radial displacement at blade tip obtained when geometrical nonlinearities are taken into account and when they are not is observed at steady-state. In this figure, 6 revolutions are simulated with a time step of $h=10^{-7} \mathrm{~s}$.

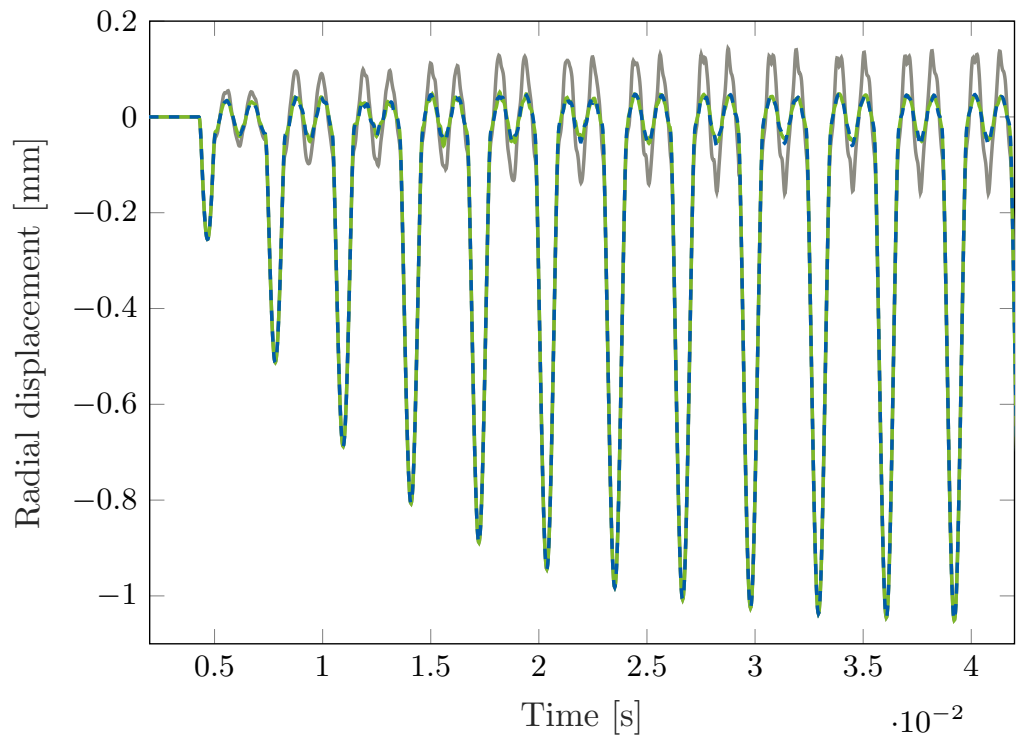

Figure 8. Evolution of the radial displacement at blade tip. Comparison of the solution obtained with the Craig-Bampton method $\left(r_{c}=15\right)$ by neglecting the geometrical nonlinearities $(-)$ and the solutions obtained with the Craig-Bampton method for different numbers $r_{c}$ of fixed interface modes $\left(r_{c}=15(-)\right.$ and $\left.r_{c}=20(--)^{\prime}\right)$ without neglecting the geometrical nonlinearities. 
The different nonlinear reduction methods are used to predict the evolution of the displacement due to contact interactions. As a first step, convergence studies are performed in order to select the number of modes to be included in the reduction bases. For the Craig-Bampton reduction method with POD filtering, for instance, Fig. 8 shows that the predicted solution does not significantly vary when the number of fixed interface linear modes is increased beyond $r_{c}=15$. Similar analyses are performed for the modal derivative reduction method and the POD reduction method.

Figure 9 compares the evolutions of the radial displacement predicted with the three different nonlinear reduction methods studied in this paper. The characteristics of the reduction bases are defined according to the convergence analyses performed. The Craig-Bampton reduction basis is composed of $r_{c}=15$ fixed interface linear modes. For the modal derivative approach, 5 internal modes and the corresponding 32 modal derivatives are included in the basis. This leads to a basis size of 40 , i.e. more than two times the basis size required with the Craig-Bampton method. Contrary to the POD bases built to study the structure in the absence of contact interaction, the POD basis is here built by considering a harmonic training simulation in the directions $\mathbf{e}_{x}$ and $\mathbf{e}_{y}$ (see Fig. 1) to be closer to the actual spatial shape of the contact excitation. $r_{\mathrm{POD}}=20 \mathrm{POD}$ modes are included in the reduction basis. A good correspondence is observed between the three displacement predictions. The three reduction methods predict similar radial displacement amplitudes in this nonlinear context. The modal derivative reduced model predicts a richer harmonic content.

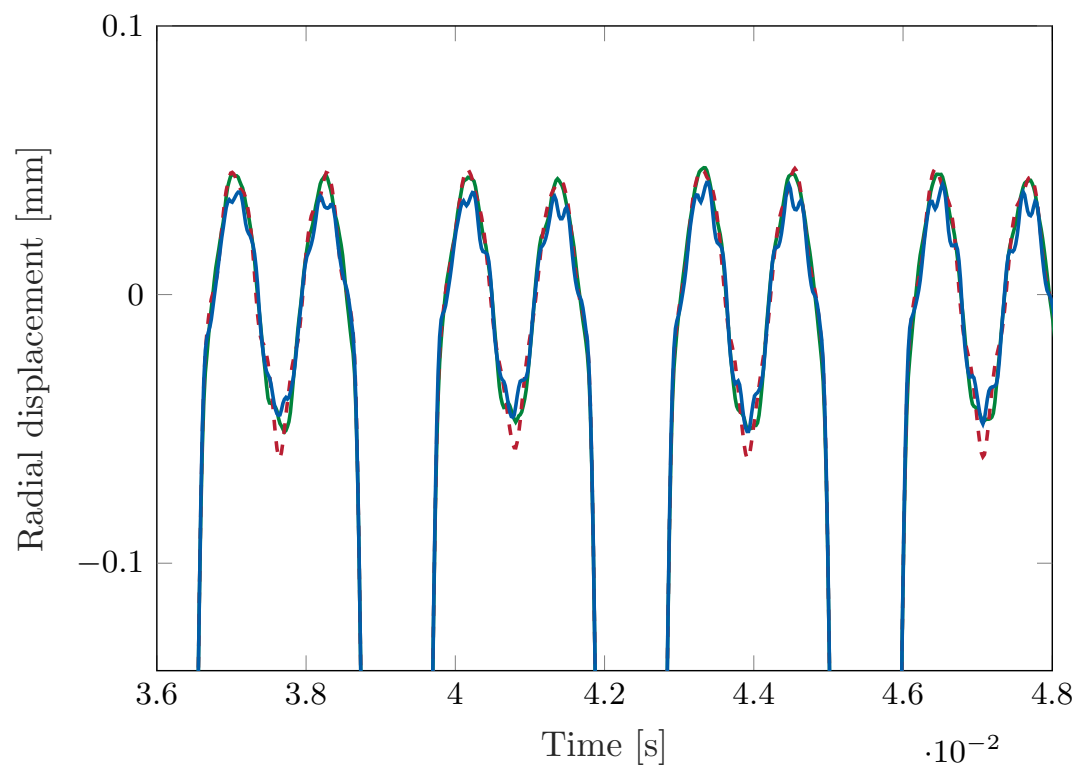

Figure 9. Evolution of the radial displacement at blade tip. Comparison of the solutions obtained with the different reduction methods accounting for geometrical nonlinearities (POD with $r=20(-)$ ), Craig-Bampton method with $r_{c}=15(---)$, modal derivatives with $r_{c}=5$ and $\left.r_{\mathrm{MD}}=32(-)\right)$.

\section{Conclusion}

The application of different nonlinear reduction methods to an industrial compressor blade undergoing large displacements and contact interactions allows to highlight the main advantages and drawbacks of the different methods. The performance and the robustness of the different reduction methods are assessed with a performance indicator combining global and local criteria. The study shows that the reduction method by Proper Orthogonal Decomposition should be preferred when the spatial shape of the external excitation is a priori known. The modal derivative approach allows to obtain slightly better results than the Craig-Bampton method with POD filtering but 
requires a larger reduction basis to get accurate results. In particular, in the case of contact interactions, a large number of modal derivatives is required to achieve the same degree of accuracy as the Craig-Bampton method.

The results show that reduced order models provide a computationally efficient alternative to high fidelity finite element models for the prediction of the time response of nonlinear structures. They also allow to provide guidelines for the selection of the reduction methods for each particular application.

As a next step, the contact algorithm will be adapted with other numerical integration schemes, such as the $\alpha$-generalized methods which are more suitable for the study of nonlinear systems. Further studies will also include the application of the reduction methods to finite element models of full bladed disk with the introduction of mistuning.

\section{Acknowledgments}

E. Delhez is supported by the Fonds de la Recherche Scientifique (F.R.S.-FNRS, Belgium) which is gratefully acknowledged.

\section{References}

[1] Vakakis, A. F., 1992. "Dynamics of a nonlinear periodic structure with cyclic symmetry". Acta Mechanica, 95(1-4), pp. 197-226. doi: 10.1007/BF01170813.

[2] Grolet, A., and Thouverez, F., 2011. "Vibration Analysis of a Nonlinear System With Cyclic Symmetry". Journal of Engineering for Gas Turbines and Power, 133(2), p. 022502. doi: 10.1115/1.4001989 - oai: hal-01977265.

[3] Picou, A., Capiez-Lernout, E., Soize, C., and Mbaye, M., 2019. "Mistuning analysis of a detuned bladed-disk with geometrical nonlinearities". In Proceedings of the ASME Turbo Expo 2019. doi: 10.1115/GT2019-90820 oai: hal-02175580.

[4] Sakulkaew, S., Tan, C. S., Donahoo, E., Cornelius, C., and Montgomery, M., 2013. "Compressor efficiency variation with rotor tip gap from vanishing to large clearance". Journal of Turbomachinery, 135(3). doi: $10.1115 / 1.4007547$.

[5] Piollet, E., Nyssen, F., and Batailly, A., 2019. "Blade/casing rubbing interactions in aircraft engines: Numerical benchmark and design guidelines based on NASA rotor 37". Journal of Sound and Vibration, 460, p. 114878. doi: 10.1016/j.jsv.2019.114878 - oai: hal-02281666.

[6] Nyssen, F., Tableau, N., Lavazec, D., and Batailly, A., 2020. "Experimental and numerical characterization of a ceramic matrix composite shroud segment under impact loading". Journal of Sound and Vibration, 467, p. 115040. doi: 10.1016/j.jsv.2019.115040 - oai: hal-02378746.

[7] Craig, R. R., and Chang, C.-J., 1976. "Free-interface methods of substructure coupling for dynamic analysis". AIAA Journal, 14(11), pp. 1633-1635. doi: 10.2514/3.7264.

[8] Seshu, P., 1997. "Substructuring and Component Mode Synthesis". Shock and Vibration, 4(3), pp. 199-210. doi: 10.3233/SAV-1997-4306.

[9] Craig, R. R., 2000. "Coupling of substructures for dynamic analyses: An overview". In Collection of Technical Papers - AIAA/ASME/ASCE/AHS/ASC Structures, Structural Dynamics and Materials Conference, Vol. 5, AIAA, pp. 3-14. doi: 10.2514/6.2000-1573.

[10] Balmaseda, M., Jacquet-Richardet, G., Placzek, A., and Tran, D.-M., 2019. "Reduced order models for nonlinear dynamic analysis with application to a fan blade". In Proceedings of ASME Turbo Expo 2019. doi: 10.1115/GT2019-90813 - oai: hal-02396583.

[11] Di Palma, N., Martin, A., Thouverez, F., and Courtier, V., 2019. "Nonlinear Harmonic Analysis of a Blade Model Subjected To Large Geometrical Deflection and Internal Resonance". In Proceedings of ASME Turbo Expo 2019. doi: 10.1115/GT2019-91213 - oai: hal-02159855. 
[12] Kerschen, G., Golinval, J.-C., Vakakis, A. F., and Bergman, L. A., 2005. "The method of proper orthogonal decomposition for dynamical characterization and order reduction of mechanical systems: An overview". Nonlinear Dynamics, 41(1-3), pp. 147-169. doi: 10.1007/s11071-005-2803-2.

[13] Weeger, O., Wever, U., and Simeon, B., 2016. "On the use of modal derivatives for nonlinear model order reduction". International Journal for Numerical Methods in Engineering, 108, pp. 1579-1602. doi: 10.1002/nme.5267.

[14] Wu, L., and Tiso, P., 2016. "Nonlinear model order reduction for flexible multibody dynamics: a modal derivatives approach". Multibody System Dynamics, 36(4), pp. 405-425. doi: 10.1007/s11044-015-9476-5.

[15] Joannin, C., Chouvion, B., Thouverez, F., Ousty, J.-P., and Mbaye, M., 2017. "A nonlinear component mode synthesis method for the computation of steady-state vibrations in non-conservative systems". Mechanical Systems and Signal Processing, 83, pp. 75-92. doi: 10.1016/j.ymssp.2016.05.044 - oai: hal-01389699.

[16] Joannin, C., Thouverez, F., and Chouvion, B., 2018. "Reduced-order modelling using nonlinear modes and triple nonlinear modal synthesis". Computers and Structures, 203, pp. 18-33. doi: 10.1016/j.compstruc.2018.05.005 oai: hal-01800394.

[17] Capiez-Lernout, E., Soize, C., and Mignolet, M. P., 2012. "Computational stochastic statics of an uncertain curved structure with geometrical nonlinearity in three-dimensional elasticity". Computational Mechanics, 49(1), pp. 87-97. doi: 10.1007/s00466-011-0629-y - oai: hal-00684289.

[18] Touzé, C., Vidrascu, M., and Chapelle, D., 2014. "Direct finite element computation of non-linear modal coupling coefficients for reduced-order shell models". Computational Mechanics, 54(2), pp. 567-580. doi: 10.1007/s00466-014-1006-4 - oai: hal-00955582.

[19] Chaturantabut, S., and Sorensen, D. C., 2009. "Discrete Empirical Interpolation for Nonlinear Model Reduction". In Proceedings of the 48th IEEE Conference, pp. 4316-4321. doi: 10.1109/CDC.2009.5400045.

[20] Muravyov, A., and Rizzi, S., 2003. "Determination of nonlinear stiffness with application to random vibration of geometrically nonlinear structures". Computers and Structures, 81(15), pp. 1513-1523. doi: 10.1016/S00457949(03)00145-7.

[21] McEwan, M. I., Wright, J. R., Cooper, J. E., and Leung, A. Y., 2001. "A combined modal/finite element analysis technique for the dynamic response of a non-linear beam to harmonic excitation". Journal of Sound and Vibration, 243(4), pp. 601-624. doi: 10.1006/jsvi.2000.3434.

[22] Sinha, S. K., 2013. "Rotordynamic analysis of asymmetric turbofan rotor due to fan blade-loss event with contactimpact rub loads". Journal of Sound and Vibration, 332(9), pp. 2253-2283. doi: 10.1016/j.jsv.2012.11.033 oai: hal-01555281.

[23] Carpenter, N. J., Taylor, R. L., and Katona, M. G., 1991. "Lagrange constraints for transient finite element surface contact". International Journal for Numerical Methods in Engineering, 32(1), pp. 103-128. doi: 10.1002/nme.1620320107 - oai: hal-01389918.

[24] Ma, H., Wang, D., Tai, X., and Wen, B., 2017. "Vibration response analysis of blade-disk dovetail structure under blade tip rubbing condition". Journal of Vibration and Control, 23(2), pp. 252-271. doi: $10.1177 / 1077546315575835$.

[25] Batailly, A., and Legrand, M., 2015. "Unilateral contact induced blade/casing vibratory interactions in impellers: Analysis for flexible casings with friction and abradable coating". Journal of Sound and Vibration, 348, pp. 344-364. doi: 10.1016/j.jsv.2015.03.027 - oai: hal-01222732.

[26] Almeida, P., Gibert, C., Thouverez, F., Leblanc, X., and Ousty, J.-P., 2016. "Numerical Analysis of Bladed Disk-Casing Contact with Friction and Wear". Journal of Engineering for Gas Turbines and Power, 138(12). doi: $10.1115 / 1.4033065$.

[27] Capiez-Lernout, E., Soize, C., and Mbaye, M., 2014. "Geometric nonlinear dynamic analysis of uncertain structures with cyclic symmetry. Application to a mistuned industrial bladed disk". In Proceedings of the 26th International Conference on Noise and Vibration Engineering, pp. 4481-4494. oai: hal-01066543. 
[28] Givois, A., Grolet, A., Thomas, O., and Deü, J. F., 2019. "On the frequency response computation of geometrically nonlinear flat structures using reduced-order finite element models". Nonlinear Dynamics, 97, pp. 1747-1781. doi: 10.1007/s11071-019-05021-6 - oai: hal-02289755.

[29] Craig, R. R., and Bampton, M., 1968. "Coupling of substructures for dynamic analyses". AIAA Journal, 6(7), pp. 1313-1319. doi: 10.2514/3.4741 - oai: hal-01537654.

[30] Tiso, P., 2011. "Optimal second order reduction basis selection for nonlinear transient analysis". In Proceedings of the International Modal Analysis Conference (IMAC) XXIX, pp. 27-39. doi: 10.1007/978-1-4419-9299-4_3.

[31] Wu, L., Tiso, P., and van Keulen, F., 2016. "A modal derivatives enhanced Craig-Bampton method for geometrically nonlinear structural dynamics". In Proceedings of the 27th International Conference on Noise and Vibration Engineering, pp. 3615-3624.

[32] Tiso, P., 2011. "Effective modal derivatives based reduction method for geometrically nonlinear structures". In Proceedings of ASME 2011 International Design Engineering Technical Conferences \& Computers and Information in Engineering Conference (IDETC/CIE 2011), pp. 399-406. doi: 10.1115/DETC2011-48315.

[33] Reid, L., and Moore, R. D., 1978. Design and Overall Performance of Four Highly-Loaded, High Speed Inlet Stages for an Advanced High-Pressure Ratio Core Compressor. Tech. rep., NASA TP 1337. URL: https://ntrs.nasa.gov/citations/19780025165.

[34] Moore, R. D., and Reid, L., 1980. Performance of Single-Stage Axial-Flow Transonic Compressor With Rotor and Stator Aspect Ratios of 1.19 and 1.26, Respectively, and With Design Pressure Ratio of 2.05. Tech. rep., NASA TP 1659. URL: https://ntrs.nasa.gov/citations/19790001889.

[35] Ameri, A. A., 2009. "NASA ROTOR 37 CFD CODE validation Glenn-HT code". In 47th AIAA Aerospace Sciences Meeting including the New Horizons Forum and Aerospace Exposition. doi: 10.2514/6.2009-1060. 